\title{
History of Radiation Therapy Technology
}

\author{
Hyun Do Huh ${ }^{1 \oplus}$, Seonghoon $\mathrm{Kim}^{2(1)}$ \\ ${ }^{1}$ Department of Radiation Oncology, Inha University Hospital, Incheon, ${ }^{2}$ Department of Radiation Oncology, Hanyang University Medical \\ Center, Seoul, Korea
}

Received 10 August 2020

Revised 2 September 2020

Accepted 2 September 2020

Corresponding author

Seonghoon Kim

(dochokim@gmail.com)

Tel: 82-2-2290-8627
Here we review the evolutionary history of radiation therapy technology through the festschrift of articles in celebration of the 30th anniversary of Korean Society of Medical Physics (KSMP). Radiation therapy technology used in clinical practice has evolved over a long period of time. Various areas of science, such as medical physics, mechanical engineering, and computer engineering, have contributed to the continual development of new devices and techniques. The scope of this review was restricted to two areas; i.e., output energy production and functional development, because it is not possible to include all development processes of this technology due to space limitations. The former includes the technological transition process from the initial technique applied to the first model to the latest technique currently used in a variety of machines. The latter has had a direct effect on treatment outcomes and safety, which changed the paradigm of radiation therapy, leading to new guidelines on dose prescriptions, innovation of dose verification tools, new measurement methods and calculation systems for radiation doses, changes in the criteria for errors, and medical law changes in all countries. Various complex developments are covered in this review. To the best of our knowledge, there have been few reviews on this topic and we consider it very meaningful to provide a review in the festschrift in celebration of the 30th anniversary of the KSMP.

Keywords: History, Korean Society of Medical Physics, Therapy machine

\section{Introduction}

History is like a mirror that can be used to guide evaluation of the present and it can illuminate the future through reflection on the past. Lessons from the past can direct Korean medical physicists and others on the way forward to develop new radiation therapy technology. Here we review changes in radiation therapy technology used worldwide to add to the festschrift of articles in celebration of the 30th anniversary of the Korean Society of Medical Physics (KSMP). We only included contents covered in the book published in memory of the 20th anniversary of the KSMP foundation, which referred to both domestic and foreign developments.

It has been 125 years since W. C. Roentgen, a German physicist, discovered X-rays in late 1895. The X-ray generating methodology was applied to cancer therapy soon after the historical discovery. Low $\mathrm{kV}$ energy was used initially and today MV X-ray technology is commonly used in clinical practice. Due to space limitations, we limited discussion on certain topics. Here we focus only on electron-accelerating X-ray machines. Particle therapy devices have been developed but this topic is beyond the scope of this article. In general, both hardware and software would be included in a history of radiation therapy technology, but software will not be discussed here and only hardware will be reviewed. 
We have included the interesting developmental history of important hardware components in medical linear accelerators (LINACs).

Radiation therapy machines currently used in clinical practice are the result of evolution over a long period of time. Various areas of science, such as medical physics, mechanical engineering, and computer engineering, have contributed to the continual development of the technology. The scope of the review was restricted to two areas; i.e., output energy production and functional development, due to space limitations. The former includes the technological transition from initial techniques applied to the first model to the latest technology that is currently in use in a variety of machines. The latter have had a direct effect on treatment outcomes and the safety of radiation therapy. These developments changed the paradigm of radiation therapy, leading to new guidelines on dose prescriptions, innovation of dose verification tools, new measurement methods and calculation systems for radiation doses, criteria for errors, and changes in medical laws in each country.

Various complex developments were covered as much as possible. To the best of our knowledge, there have been few reviews of these topics and we consider it very meaningful to provide a review in the festschrift in celebration of the 30th anniversary of the KSMP foundation.

\section{Changes in radiation therapy}

1) Discovery of $X$-rays and the beginning of radiation therapy

Radiotherapy can be traced back about 125 years to the discovery of X-rays (1895) by a Germany physicist named W. C. Roentgen. After X-rays were discovered, it did not take long before X-rays were used in cancer treatments. On January 29, 1896, just three days after announcement of the discovery of X-rays, the E. H. Grubb company, a vacuum tube manufacturer, applied X-rays for the first time in cancer treatment at the suggestion of doctor Ludlam [1,2]. A single treatment was performed for about one hour in breast cancer patients. In 1900, T. Stenbeck and T. Sjogren treated patients with skin cancer at the tip of their nose [3]. In 1903, Senn [4] first attempted treatment in a leukemia patient. In 1896, Despeignes began using radiotherapy in
France. Patients with stomach cancer were irradiated 15 to 30 minutes with 80 fractions and it was reported that the disease improved and pain was relieved [1].

In 1902, Guido Holzknecht of Austria initiated the first proposal for Roentgen-based dosimetry. When his chromoradiometer was exposed to X-rays, a salt mixture changed from yellow to green. The levels of change with various radiation doses were compared to chromoradiometer readings calibrated in Holznecht $(\mathrm{H})$ units [5]. The use of an ionization chamber was then adopted in 1928 at the first Association of International Radiology conference held in Stockholm, Sweden [6]. Initial radiation therapy relied on low energy with significantly low penetration power. There was neither an exact concept of the radiation dose nor the location of the tumor. Moreover, there was no concept of treatment planning as is used today. Since then, Co-60 beam therapy emitting megavoltage gamma rays became the common treatment in most radiotherapy departments in the 1950 s to 1960 s and this led to much research. This time period has become known at the first radiotherapy period. Only in the middle of the 20th century were exact dosimetry systems developed [7].

\section{2) Introduction of radiotherapy devices to Korea}

It is difficult to obtain exact information on when radiotherapy devices were first introduced to Korea, but the book of "Past Footprints" released by the Department of Radiation Oncology, Yonsei University College of Medicine, reports 1937 as the first year. The equipment at that time was an X-ray generating device with a combined use of diagnosis and treatment. It had a brand name of Special Polex and was produced by the Shimazu Japanese Company. The system was characterized by a tube voltage of $200 \mathrm{kVp}$, tube current of $20 \mathrm{~mA}$, and $10 \mathrm{~Gy}$ emitting power (Fig. 1) [8]. The book describing the 20 year history of the Department of Radiation Oncology of Chonnam National University Hospital reported that a radiation oncology class was founded in 1953 and it took over the superficial cancer treatment machine (uncontrollable gas tube, Polyphos, Siemens, 135 $\mathrm{kVp} / 5 \mathrm{~mA}$, which doubled as diagnostic equipment) from the provincial hospital, predecessor of Chonnam National University Hospital, but it is unknown whether radiotherapy was actually performed. The book published in memory 


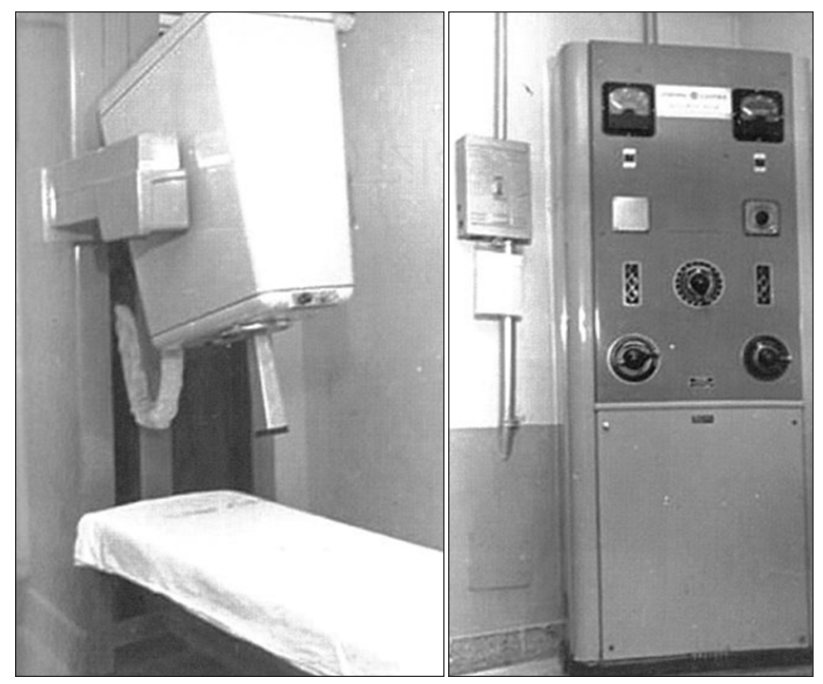

Fig. 1. Deep-seated cancer therapy machine with $250 \mathrm{kVp}(250$ kVp, 15 mA, Model: GE Maximar 250-III) installed at Severance Hospital in 1961 [8].

of the 30th anniversary of the Radiation Oncology Department of Catholic University Hospital reports that it installed a Maximar (50 kVp and $20 \mathrm{~mA}$ ) in 1960 and began offering radiation therapy.

Since then, medical LINAC machines with megavoltage high energy were introduced in Korea through pioneering passion and endeavors even in poor surroundings. Severance Hospital was the first to purchase a clinical LINAC machine in Korea. The book of "Past Footprints" mentioned above reports that a high energy LINAC (LMR-13, Toshiba) was first introduced in Korea in 1972 at Severance Hospital with the assistance of the Japanese Overseas Technical Cooperation Group (OTCA). The LMR-13 was an electron accelerator that adopted a traveling wave mode and produced electron energies of $8 \mathrm{MeV}, 10 \mathrm{MeV}$, and $12 \mathrm{MeV}$, X-ray energy of $13 \mathrm{MV}$, and a dose rate of 400-600 rad. At that time, it was thought that the machine had excellent performance because the X-ray beam or electron beam could be chosen according to the patient's needs. Following Severance Hospital's introduction of LINACs, there are no accurate records of another introduction of high energy LINACs in Korea in the 1970s. About 10 years later, St. Mary's Hospital in Seoul introduced a 6 MV high energy LINAC in 1983 and the Department of Radiation Oncology, Korea Atomic Energy Hospital, introduced Micron accelerators with X-ray energies of 6MV, $10 \mathrm{MV}$, and $21 \mathrm{MV}$ in 1985. The book pub- lished in memory of the 30th anniversary of the Radiation Oncology Department, Seoul National University Hospital, recorded that it started radiation therapy by introducing a Clinac 6/10 (Varian Company, Palo Alto, CA, USA) in 1985.

The Cobalt teletherapy machine was used for radiation therapy before the introduction of a clinical LINAC. Since the 1980s, high energy LINACs were introduced nationwide for radiation therapy. Dr. Ji Young-Hoon, who worked at the Korea Atomic Energy Hospital, reported in his documents on the "present development status and prospect for radiation therapy machine of Korea" that high energy LINACs, as many as about 200, were introduced and used in the clinical institution as of 2017, including Cyberknife and Tomotherapy. Needless to say, this number is fewer than that of Europe or the Americas, but the quality of LINAC machines and treatment levels in Korea does not suffer by comparison with those of any medically advanced countries. High level radiation therapy machines have been introduced and used in clinical practice, but professionals such as medical physicists who work with such therapy machines are very few compared to Europe or the Americas.

3) Application of computational tomography to radiation therapy

Computed tomography (CT) was not applied, as it is today, in radiation therapy during the days when Co-60 and the initial model of a megavoltage LINAC were used in clinical applications. Treatment planning was performed manually by an expert with related knowledge using radiographic images of cross sections of the tumor center or anatomical center of the treatment site. As for the application of radiation imagery to radiotherapy, single photon emission computed tomography and positron emission tomography emerged in the 1960s but were not applied for radiation treatment planning. CT came into wide use around 1972 and started to be utilized in radiation treatment planning. In 1960, treatment plans using radiation images started and 2-dimentional treatment planning was carried out for a short time until CT was used. Treatment planning did not achieve significant progress until patient-specific 3-dimentional (3D) treatment planning began to be implemented. Since then, personal computers began to be widely used and led to remarkable progress in radiation treatment plan- 
ning using radiation images. At this time, many researchers studied treatment planning related topics and produced many reports [9].

It was very difficult to get good information on when computed treatment planning systems were introduced in Korea. The book of "Past Footprints" by the Radiation Oncology Department of Yonsei University College of Medicine records that modern treatment planning could be implemented in August 1970 only after the introduction of measurement devices capable of measuring and plotting isodose distribution curves. Prior to the introduction of these systems, treatment plans were implemented manually. Since 1980, high energy LINACs were widely introduced and then computed treatment planning systems were also introduced. In 1994, computed treatment planning systems capable of 3D treatment planning were introduced.

\section{4) Paradigm shift in radiation therapy}

(1) Start and development of intensity modulated radiation therapy

Brahme, the Swedish medical physicist, introduced Intensity Modulated Radiation Therapy (IMRT) in 1982 [10]. He proposed to change the present shape of a collimator into that of a multileaf collimator (MLC) and then the Scandtronics Company implemented it. The NOMOS Mimic system was commercially released in 1992. It first started at Baylor in 1994 and it took about three years to implement modulated radiation therapy using techniques developed by the Memorial Sloan Kettering Cancer Institute in 1997 [11-13]. The idea of MLC delivering IMRT from a step and shoot mode or dynamic mode was not clinically established before 1997. Actual IMRT was applied to clinical practice only in 2000. Although IMRT relied on Nomos Mimic or MLC-using technology, these two techniques began simultaneously in 1992 and have been developing independently.

The Radiation Oncology Department at Hallym University Sacred Heart Hospital, began using IMRT in Korea with a Siemens Primus LINAC that had a double focus 54leaf MLC purchased in August 2000. Cho, who is the current president of the KSMP, established the first guidelines for physical data in Korea, quality assurances, and quality assurances of patient treatment so that IMRT could be car- ried out successfully [14]. IMRT was first performed on a prostate cancer case and successfully implemented. As the national medical insurance policy was extended in favor of IMRT as of 2020, all of the radiation oncology departments in Korea implement IMRT.

(2) Birth of new types of radiotherapy machines

With the introduction of MLC, a new concept of radiotherapy devices based on the existing C-type of LINAC was developed and supplied. Tomotherapy, which applied such a new concept of technology, was launched in 2002 [14]. The first tomotherapy device in Korea was installed in November 2005 at Incheon St. Mary's Hospital. About 27 tomotherapy devices are installed and have been operating as of 2020. In 2017, the Varian Company launched Halcyon, which had a similar function to tomotherapy, which was first installed at Pittsburg University Hospital and, in Korea, it was first installed and used in clinical practice at Korea University Anam Hospital in late 2018.

\section{(3) Image guided radiotherapy}

In recent years, the rapid development of radiotherapy has led to progress in radiotherapy simultaneously in another aspect. Systems were developed to consider and verify the internal and interstitial displacements of the patient's organs during treatment. Cyberknife, which was first devised by John R. Adler, a neurosurgeon at Stanford University, together with Swedish Peter and Russell Schonberg of Schonberg Research Center, is a typical machine capable of tracking the position of the tumor in real time. This machine was the first with a LINAC system that was capable of delivering a radiation beam in 3D space. It was first installed at Stanford University Hospital, USA in 1991, and was permitted from the United States Food and Drug Administration to be applied to head and neck cancer in 1999 and extended to whole body treatment in 2001.

It was first introduced in Korea and used for radiation surgery and treatment at the Korea Cancer Center Hospital in 2002. This machine was developed multiple times, resulting in the release of the G3 model in 2005, which was capable of tracking tumor motion with the patient's breathing in real time. The more enhanced G4 models were installed in Korea at Soon Chun Hayng University Cheonan 
Hospital and Inha University Hospital in 2008. The model M6 equipped with a mini MLC was released in 2012 and installed at Severance Hospital Cancer Center. Recently, conventional LINAC machines were also equipped with the techniques to track tumor movement and use the gating technique to treat tumors during delivery of IMRT. Good cooperation from many different fields, such as medicine, medical physics, radiology, computer engineering, and so on, have led to the development of radiotherapy with the result of remarkable progress in today's radiation treatment techniques.

\section{Birth of radiation cancer therapy devices and development of high energy devices}

\section{1) Initial model of radiation cancer therapy devices}

Current cancer therapy includes radiotherapy using an $\mathrm{X}$-ray beam. Treating cancer using a medical LINAC has become a main trend in cancer therapy. Clinical LINAC models currently used commercially in clinical practice have evolved continuously through many trials and errors. Until 1950, external beam radiotherapy has been performed mainly with X-rays generated at a maximum of $300 \mathrm{kV}$. In the1950s and 1960s, the existing $\mathrm{kV}$ machines were replaced gradually with high energy machines as the development of high energy photon-emitting accelerators and Co-60 therapy machines became highly popular. Nevertheless, $\mathrm{kV}$ machines with low energy beams did not fade away until recently and have existed for the treatment of special sites.

In this chapter, we review X-ray generators that were the starting point of high energy machine development and were used for the first radiotherapy procedures. They predicted the kind of machines that would be developed in the future. The Radiotherapeutic Research Unit of the Medical Research Council at Hammersmith Hospital, London, installed the first clinically usable high energy LINAC. It was used February 1953 for physics experiments and other tests. Patient treatment was attempted on September 7 the same year but it was not used for patient treatment exclusively [15]. The Granz Ray Therapy device, which generated $\mathrm{X}$-rays of less than $20 \mathrm{kV}$, was the first machine to be used for treatment before high energy X-ray generating LINAC devices. Contact Therapy generated X-rays in the range of
$40-50 \mathrm{kV}$ and it was used to treat patients with contact close to the treatment lesion. This therapy was suited for superficial tumors because the maximum absorbed dose was near the skin. Superficial Therapy machines were developed next. They generated X-rays in the range of 50-150 kV and were applied for radiotherapy. Orthovoltage Therapy and Deep Therapy that generated energies of $150-500 \mathrm{kV}$ were developed and utilized for cancer treatment. This system had a source-surface distance (SSD) of about $50 \mathrm{~cm}$ and used cones to collimate the beam to the desired size. It still had a maximum dose near the skin but $90 \%$ of the dose occurred at a depth of about $2 \mathrm{~cm}$ below the skin, which facilitated treating the tumor deeper than with Superficial Therapy. Fig. 2a shows graphs of the percentage depth dose for Granz to Orthovoltage with different beam qualities and maximum doses at the skin surface, while Cobalt-60 (Co-60) had its maximum dose at some distance below the skin and much less skin reaction than with the $\mathrm{kV}$ machines. The device (Fig. 2b) is a superficial therapy machine equipped with a cone. Fig. 2c shows a superficial skin cancer that was treated with this type of machine.

However, there have been problems when the skin was exposed to large radiation doses to treat deep-seated tumors. From the 1950s to the 1960s, many efforts were made to develop a device capable of generating high energy photon beams. Using a step just before high energy, Supervoltage Therapy was developed that could generate up to 1,000 kV. The Van De Graff Generator without Co-60 teletherapy was the first to produce MV high energy. This system was an electrostatic accelerator designed to accelerate charged particles. This machine accelerated electrons to produce high energy X-ray beams of up to $2 \mathrm{MV}$ in radiation therapy. This machine is not commercially produced anymore because Co-60 teletherapy machines and medical LINACs with better technical and physical advantages came to be used.

\section{2) Development of hospital-dedicated high energy LINACs}

The need for high-energy radiotherapy machines was recognized from the beginning of radiotherapy, but technical shortages hampered development. In 1953, MetropolitanVickers contributed to the advent of LINACs. He developed 
a

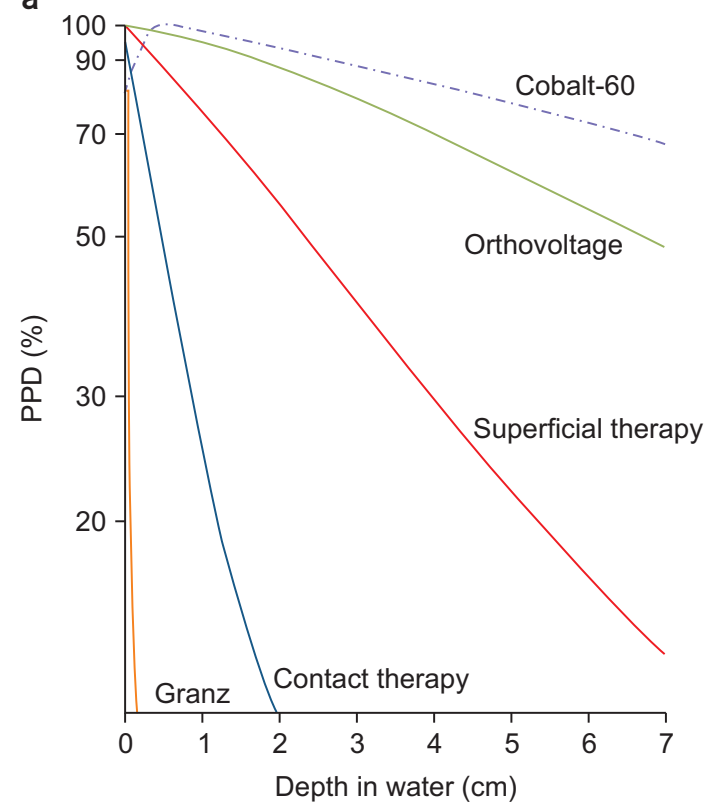

b

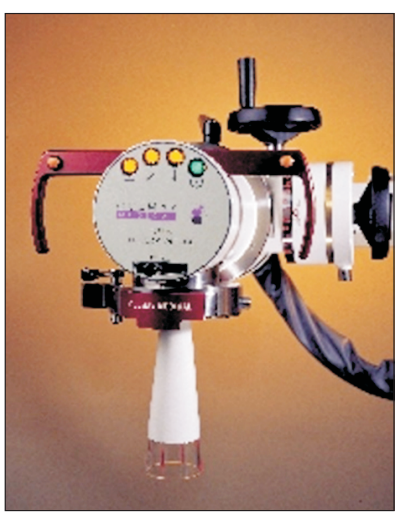

C

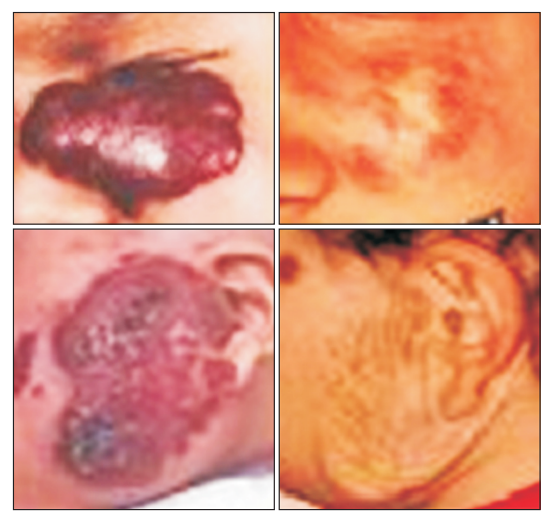

Fig. 2. The developmental process of $\mathrm{kV}$ therapy machines from Granz to Orthovoltage. (a) The graph shows percentage depth doses (PDDs) of $\mathrm{kV}$ X-ray therapy machines and Co-60 machines with the maximum dose at a distance below the skin. (b) The machine in picture is a superficial therapy machine equipped with a cone, and (c) the picture demonstrates that superficial skin cancer can be treated using this type of machine.

and produced a LINAC device that accelerated charged particles, such as electrons, to high energies through linear a vacuum tube using high-frequency electromagnetic waves. This LINAC produced 8 MV X-rays using 3 meter acceleration or corrugated waveguides, was used to treat patients, and was the only LINAC machine at that time [16]. This was immediately followed by the additional installation of two LINACs using $4 \mathrm{MV}$.

One of them was installed at Newcastle by Mullard Equipment Ltd, a division of Philips, and the other was installed at Manchester Christie Hospital by Met-Vic. In addition, Met-vic supplied two more in 1955. They were installed at Western General Hospital, Edinburgh, and Mount Vernon Hospital, Middlesex [17]. In addition, a Stanford University research group in California, USA installed a 6 MV LINAC at Stanford University in the Department of Radiology in 1954. They treated the first patient in 1956. At that time, seven LINACs were installed through the world. The $15 \mathrm{MV}$ machines were installed at St. Bartholomew's Hospital and a few medical centers in Chicago. At that time, these machines were said to be installed or under installation, but it was not clear whether they had been applied in clinical practices $[17,18]$.
Three Met-Vic systems were operating in Australia from 1956 to 1957 and, not long after that, they were installed in New Zealand as well. Until 1962, the Mullard Company installed LINAC systems in Australia, Japan, and Russia. Varian Company installed the first prototype of a $6 \mathrm{MV}$ isocenter LINAC at University of California, Los Angeles Medical Center. A Mevatron was installed in France in 1965 [17]. Since then, high energy LINACs have been evolving. The development of high energy radiotherapy machines has a deep connection with that of electron beam acceleration modes.

\section{3) Historical origin of a high energy LINAC}

In 1928, the mode of electron acceleration, proposed by Ising, a Swedish physicist, and developed by Wideroe, a Norwegian physicist, connected a series of linear tubes that allowed electrons to gain energy whenever they went through the gaps (Fig. 3). This principle had a limitation for clinical use in that the length of the accelerator tube increased with electron speed when the frequency was kept constant.

In the mid-1930s, Hansen from Stanford, CA, USA, developed an electron accelerating machine called Rhumbatron. 


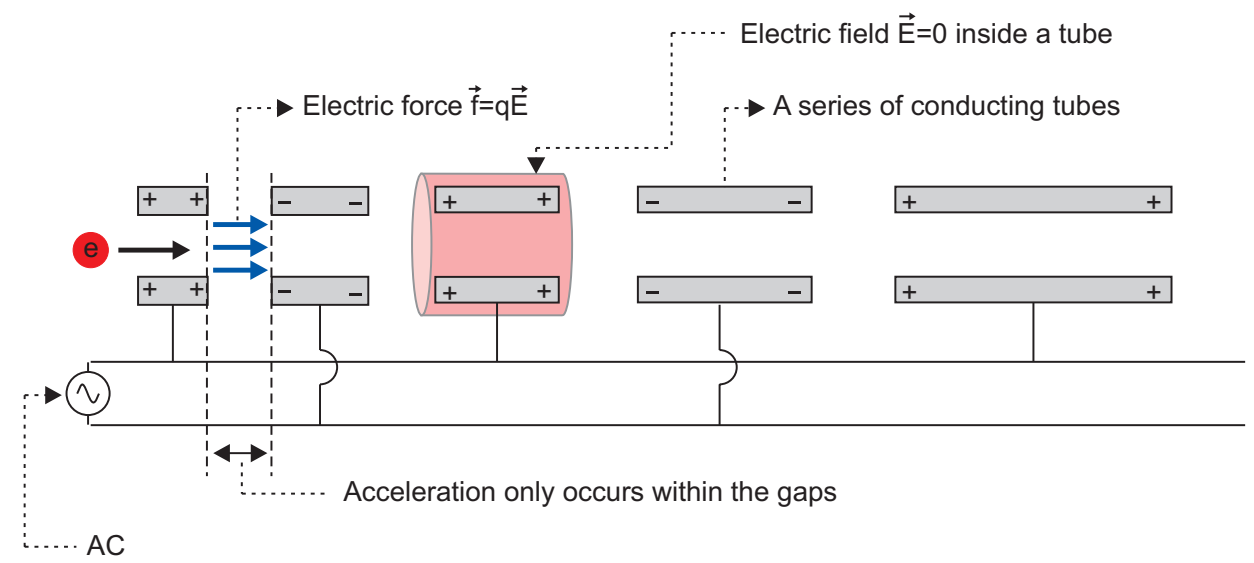

Fig. 3. Initial design of the electron acceleration mode. Electrons were drifted through a series of flight tubes connected to an alternating voltage supply (AC) and accelerated only as they passed through the gaps between the drift tubes.
This device repeatedly passed electrons through a resonant microwave cavity to increase energy when passing each section [19]. However, the electric power level at that time from the microwave generator was inappropriate for clinical applications. Through World War II, war radar devices in the military required a microwave generator with MW output, and two tubes with this type of performance were developed. In 1939, Boot and Randall [20] (England) designed the Magnetron, and in 1939, the Varian brothers (USA) developed the Klystron [21]. The basic difference between these two is the Magnetron is a self-generator that generates frequencies in response to DC input, while the Klystron is, in principle, an amplifier that amplifies the low power microwave input. These war radar systems were operated at a frequency of $3 \mathrm{GHz}$, which corresponds to a wavelength of $10 \mathrm{~cm}$ in free space, and they were well suited for the development of electron accelerators. Since 1945, the development of LINACs using microwaves has been done independently in the United States and the United Kingdom.

For the first time, the LINAC models were developed by adopting a traveling wave mode of acceleration modes. The LINAC, which adopted the first traveling wave mode for clinical use, was installed at Hammersmith with an accelerator tube length of about $3 \mathrm{~m}$ and a $2 \mathrm{MW}$ magnetron. The beam traveling in a horizontal direction was bent by 90 degrees to a target. It had a field size of maximum $20 \mathrm{~cm}$ and dose rate of around $150 \mathrm{rad} / \mathrm{min}$. The Varian Company, USA, at first adopted the traveling wave mode as well, but in 1962, the standing wave mode was adopted by Knapp et al. [22]. The main difference in this design was it produced an improved shunt impedance, enabling significant reduction of the accelerator size at the same energy. This design could produce 4-6 MV of energy from waveguides of 30-35 cm.

In the 1970s, LINACs were not changed dramatically in basic concept or structures, but the wedge system and the precision of the beam bending system were continuously improved. Therapy machines equipped with a general safety system were developed using the latest optimization techniques, such as a magnetron, control system, and other components. At the time of development, the advantages were not clear, but the initial therapy devices were very important in the evolution of sophisticated and modern LINACs used currently in the clinic.

Clinically available devices following physicists' research steps are the following: basic control system, safety and support system, beam correction, patient positioning accessory, beam isotropic mounting, full rotation, vacuum pump, other vacuum component development, beam bending system, energy increase, independent collimator (MLC), electronic portal imaging device (EPID), computer control, dynamic wedge, IMRT function, and IGRT system, which has become easy to use in recent years. At the same time, various, accurate, and automated patient positioning and identification systems have been developed.

As to the development of medical LINACs, dose rates are important. Medical LINACs have experienced dramatic enhancement of dose rates since their initial development. LINAC-based radiation therapy for cancer treatment began with the first patient in 1953 in London, UK, at Hammersmith Hospital, with an 8 MV machine built by Metropolitan-Vickers and installed in 1952, which was the first dedicated medical LINAC. A short while later in 1954, 
a 6 MV LINAC was installed at Stanford, CA, USA, which was used for treatments in 1956 (Fig. 4a). The first Stanford LINAC device produced output of $65 \mathrm{rad} / \mathrm{min}$ at the maximum of the transition curve in water. Conventional medical LINACs use fixed dose rates of, for example, $200 \mathrm{MU} / \mathrm{min}$ (=2 Gy/min) and $300 \mathrm{MU} / \mathrm{min}(=3 \mathrm{~Gy} / \mathrm{min})$ for $6 \mathrm{MV}$ and 10 MV, respectively, at the depth of a maximum dose in a water phantom on the central axis beam when irradiated with a $10 \times 10 \mathrm{~cm}^{2}$ field at a SSD of $100.0 \mathrm{~cm}$. The latest LINAC can produce a dose rate of up to $20 \mathrm{~Gy} / \mathrm{min}$. Maxim et al. [23] are developing pluridirectional high-energy agile scanning electronic radiotherapy (PHASER) as a platform for clinical translation of FLASH (mentioned briefly in Chapter 3) cancer radiotherapy (Fig. 4b), which requires dose rates exceeding $\sim 50 \mathrm{~Gy} / \mathrm{s}$. Such a rapid rise of dose rates reflects one aspect of the future trends of clinical LINACs.

\section{4) Development status of LINACs in Korea}

The radiation cancer therapy devices installed and used for cancer treatment in about 100 medical institutions in Korea are all imported from abroad. Many research groups in Korea have been participating in the government-led development of medical LINACs from 10 years ago to achieve technological independence.

In 2012, Dr. Young-Hoon Ji's research team at the Korea Atomic Energy Research Institute attempted to develop a Korean LINAC supported by the Ministry of Science and
ICT, and Future Planning with the aim of providing them to developing countries and Korea. They did not reach the stage of application due to a lack of research funding, but succeeded in the development of a general purpose MLC and treatment planning system. Supported by the Ministry of Knowledge Economy in 2012, professor Chae Jongseo at Sungkyunkwan University, aimed to develop a dualhead LINAC designed to reduce $30 \%$ of the treatment time through joint participation from the Korea Atomic Energy Research Institute, Yonsei University, Pusan National University, Kyungpook National University, and Korean companies.

At the same time, the team of Dr. Kyung-Min Jeong at the Korea Atomic Energy Research Institute conducted the development of a LINAC radiotherapy machine equipped with high-performance radiation for therapy and an imaging system supported by the Ministry of Science and ICT. As a segment of leading industry and promoting industry in metropolitan economic blocks in 2012, Taesung Precision Co., Ltd led the development of a high-powered X-ray and electron beam LINAC equipped with a small accelerator using an S-band for clinical applications and bio-industry in cooperation with the Atomic Energy Medical Center in the southeast of Korea. In Korea, the government-led development of LINACs has been attempted many times. Such efforts were not put to practical use because of the lack of lasting support from the government and poor participa-

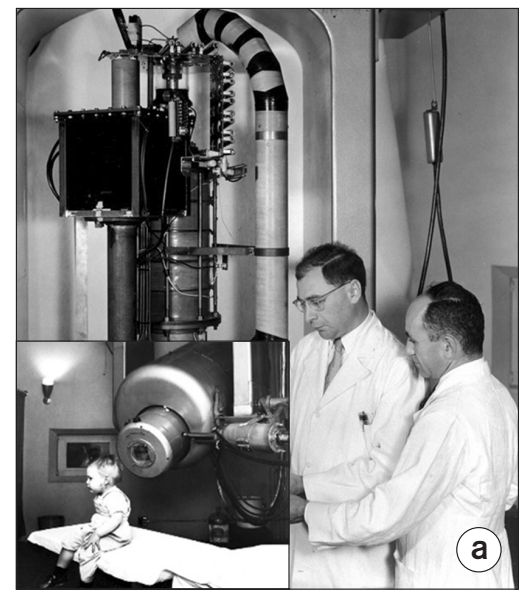

First standard medical LINAC

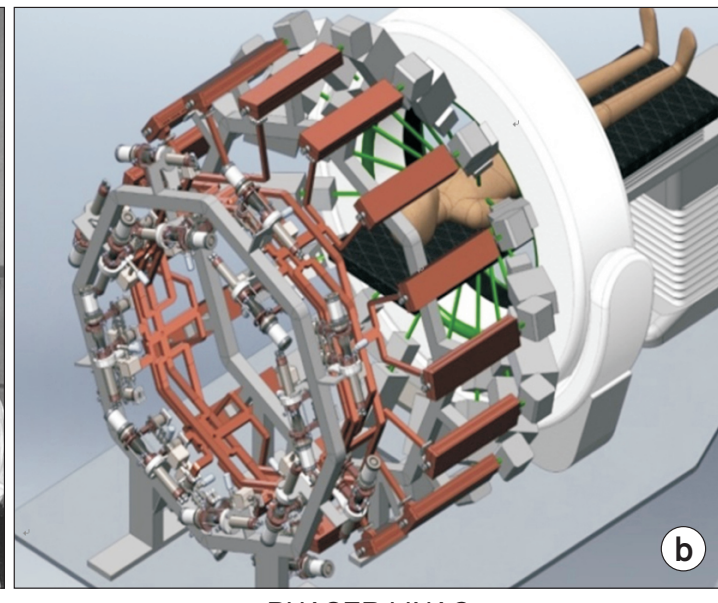

PHASER LINAC

Fig. 4. From the initial medical LINAC to a future design under development. Dr. Kaplan and physicist Ginzton developed the first 6 MV medical linear accelerator (a) in the Western hemisphere in 1953, which was used to treat a 2 year-old boy with retinoblastoma in 1956 (Stanford Report, April 18, 2017). (b) Maxim et al. are developing pluridirectional high-energy agile scanning electronic radiotherapy (PHASER), which aims to be a platform for clinical translation of FLASH cancer radiotherapy [23]. 
tion from companies, but constant interest from related professionals has been making continuous accumulation of related technologies.

\section{Future radiotherapy directions}

It is expected that, in the future, radiotherapy will be conducted in various directions. As particle therapy evolves, in addition to minimalization of the side effects of radiotherapy, the efficiency of radiotherapy will be enhanced. Proton therapy has been practiced worldwide as representative of particle therapy.

The burden of initial costs makes medical institutes hesitate to buy proton therapy devices, but it is thought that it is only a matter of time for them to be widely used due to the continuous development of technology, such as heavy particle therapy. Japan is advanced in heavy particle therapy but the introduction of such a therapy machine is in progress in Korea. Severance Hospital is moving forward to finishing installing a heavy particle therapy machine with the aim of starting cancer treatment in 2020. The region Dongnam has been working on introducing a heavy particle therapy machine in cooperation with Seoul National University Hospital. Boron-Neutron Capture therapy (BNCT) is also expected to be introduced in Korea, but no medical institutes in Korea have established installation. Some countries other than Korea have already finished development of BNCT installations in medical institutions and have started to develop a commercial version of the device.

In Korea, the Korean government has been carrying out a project in cooperation with Gachon University Gil Medical Center and Dawonmedax Company. Future radiotherapy will meet a time of diversity when patients can select any of the therapy modalities, such as X-ray, particle therapy, neutron therapy, and so. In recent years, a new paradigm of radiotherapy has been proposed. In 2014, the Vincent Favsudon group (Curie Research Center, Paris, France) reported their findings that ultrahigh dose-rate FLASH irradiation increases differential responses between normal and tumor tissues in mice and proposed a new paradigm of radiation therapy called FLASH therapy [24]. They found a very different outcome with FLASH therapy compared to current radiation therapy based on animal testing. The con- cept of FLASH therapy is to involve the ultra-fast delivery of radiation treatment at dose rates (40 to $120 \mathrm{~Gy} / \mathrm{s}$ ) several orders of magnitude greater than those currently in routine clinical practice. This therapy delivers high doses to tumors with a greater probability of tumor control and little or no normal tissue damage compared to current radiotherapy.

In 2018, the Marie-Catherine Vozenin group reported the advantages of FLASH therapy implemented on pig skin [25-27]. In 2019, the Jean Bourhis group performed FLASH therapy for the first time on patients and reported the outcome [28]. FLASH therapy has the potential power to completely change present radiotherapy and is considered to be a method of radiotherapy that can bring about a dramatical change in such many different fields, such as new therapy machines, accurate dosimetry, and understanding of biological mechanisms.

Radiotherapy in the future is expected to continue to make progress and will develop beyond what has been achieved until now. In these times of dramatically changing radiotherapy technology, both individuals and the KSMP need to make continuous progress with obtaining information and reacting to new developments.

\section{Summary}

The historical development of radiation treatment technology has been rapid. Professionals in the field need to be alert and maintain awareness of this progress. The history of radiation therapy in Korea was included here but there was a limit in this report due to the lack of documents. The history of radiation treatment devices is thoroughly that of foreign manufacturers. If the same review title is used in the future, it is expected that a history of machines developed in Korea will be included. The history of radiotherapy has been changing and developing as much as changes from manual phones to smart phones. However, there is something that cannot be changed, which is the final goal to make cancer patients $100 \%$ curable. Many technologies and devices for radiotherapy have been proposed and developed to achieve this goal. However, it is suggested that the final goal can be achieved only by educating and training more specialists, such as medical physicists, who are in step with these changes and developments. Even at the 
time of the 30th anniversary of the KSMP, we are still in the early stage of training Korean specialists in medical physics. In the future, it is expected that various techniques of radiotherapy, such as proton therapy, heavy particle therapy, BNCT, and FLASH therapy, will be widely or specially used. It is recommended that the KSMP should focus on educating and training medical physicists in order to keep up with recent developments at this turning point of radiotherapy evolution. The KSMP should achieve continuous progress toward the 40th, 50th, and 100th anniversaries to take advantage of advanced or new technologies in the future.

\section{Conflicts of Interest}

The authors have nothing to disclose.

\section{Availability of Data and Materials}

All relevant data are within the paper and its Supporting Information files.

\section{Author Contributions}

Conceptualization: Hyun Do Huh and Seonghoon Kim. Data curation: Hyun Do Huh and Seonghoon Kim. Formal analysis: Hyun Do Huh and Seonghoon Kim. Investigation: Hyun Do Huh and Seonghoon Kim. Writing-original draft: Hyun Do Huh and Seonghoon Kim. Writing-review \& editing: Hyun Do Huh and Seonghoon Kim.

\section{References}

1. Case JT, Buschke F. History of radiation therapy. Progress in radiation therapy. New York: Grune \& Stratton; 1958:1341.

2. Lederman M. The early history of radiotherapy: 1895-1939. Int J Radiat Oncol Biol Phys. 1981;7:639-648.

3. Holsti LR. Development of clinical radiotherapy since 1896. Acta Oncol. 1995;34:995-1003.

4. Senn N. Case of spleno-medullary leukemia successfully treated by use of roentgen ray. Med Rec N Y. 1903;63:281282.

5. Thames HD Jr. Early fractionation methods and the origins of the NSD concept. Acta Oncol. 1988;27:89-103.

6. Quimby EH. The history of dosimetry in roentgen therapy. Am J Roentgenol Radium Ther. 1945;54:688-703.

7. Buschek F. Radiation therapy: the past, the present, the future. Paper presented at: Fifty-first Annual Meeting of the American Radium Society; 1969 Apr 27-30; Pennsylvania, USA.p. 236.

8. Kim KE. Past Footprints. Seoul: The Department of Radiation Oncology, Yonsei University College of Medicine; 2009:46-53.

9. Webb S, Evans PM. Innovative techniques in radiation therapy: editorial, overview, and crystal ball gaze to the future. Semin Radiat Oncol. 2006;16:193-198.

10. Brahme A, Roos JE, Lax I. Solution of an integral equation encountered in rotation therapy. Phys Med Biol. 1982;27: 1221-1229.

11. Palta JR, Mackie R, Chen Z. Intensity-modulated radiation therapy- the state of the art. Madison: Medical Physics Publishing; 2003:1-23.

12. Royal College of Radiologists. Development and implementation of conformal radiotherapy in the United Kingdom. London: Royal College of Radiologist; 2002.

13. Sternick ES. The theory and practice of intensity modulated radiation therapy. Madison: Advanced Medical Publishing; 1997.

14. Cho B. Intensity-modulated radiation therapy: a review with a physics perspective. Radiat Oncol J. 2018;36:1-10.

15. Thwaites DI, Tuohy JB. Back to the future: the history and development of the clinical linear accelerator. Phys Med Biol. 2006;51:R343-R362.

16. Miller CW. An $8 \mathrm{MeV}$ linear accelerator for x-ray therapy. Manchester: Metropolitan-Vickers Electrical Co.; 1955.

17. Miller CW. Linear accelerators in clinical service. Manchester: Metropolitan-Vickers Electrical Co.; 1956.

18. Karzmark CJ, Pering NC. Electron linear accelerators for radiation therapy: history, principles and contemporary developments. Phys Med Biol. 1973;18:321-354.

19. Hansen WW. A type of electrical resonator. J Appl Phys. 1938;9:654-663.

20. Boot H, Randall JT. Historical notes on the cavity magnetron. IEEE Trans Electron Devices. 1976;23:724-729.

21. Varian RH, Varian SF. A high frequency oscillator and amplifier. J Appl Phys. 1939;10:321. 
22. Knapp EA, Knapp BC, Potter JM. Standing wave high energy linear accelerator structures. Rev Sci Instrum. 1986;39: 979-991.

23. Maxim PG, Tantawi SG, Loo BW Jr. PHASER: A platform for clinical translation of FLASH cancer radiotherapy. Radiother Oncol. 2019;139:28-33.

24. Vozenin MC, Baumann M, Coppes RP, Bourhis J. FLASH radiotherapy international workshop. Radiother Oncol. 2019;139:1-3.

25. Vozenin MC, De Fornel P, Petersson K, Favaudon V, Jaccard M, Germond JF, et al. The advantage of FLASH radiotherapy confirmed in mini-pig and cat-cancer patients. Clin Cancer Res. 2019;25:35-42.
26. Favaudon V, Caplier L, Monceau V, Pouzoulet F, Sayarath M, Fouillade C, et al. Ultrahigh dose-rate FLASH irradiation increases the differential response between normal and tumor tissue in mice. Sci Transl Med. 2014;6:245ra93.

27. Beyreuther E, Brand M, Hans S, Hideghéty K, Karsch L, Leßmann E, et al. Feasibility of proton FLASH effect tested by zebrafish embryo irradiation. Radiother Oncol. 2019; 139:46-50.

28. Skouboe S, Ravkilde T, Bertholet J, Hansen R, Worm ES, Muurholm CG, et al. First clinical real-time motion-including tumor dose reconstruction during radiotherapy delivery. Radiother Oncol. 2019;139:66-71. 